\title{
Wide-Field InfraRed Survey Telescope (WFIRST) Slitless Spectrometer: Design, Prototype, and Results
}

\author{
Qian Gong $^{\mathrm{a} *}$, David Content ${ }^{\mathrm{a}}$, Margaret Dominguez ${ }^{\mathrm{a}}$, Thomas Emmett ${ }^{\mathrm{a}}$, Ulf Griesmann ${ }^{\mathrm{b}}$, John \\ Hagopian $^{\mathrm{c}}$, Jeffrey Kruk ${ }^{\mathrm{a}}$, Catherine Marx ${ }^{\mathrm{a}}$, Bert Pasquale ${ }^{\mathrm{a}}$, Thomas Wallace ${ }^{\mathrm{a}}$, Arthur Whipple \\ ${ }^{a}$ NASA Goddard Space Flight Center, 8800 Greenbelt Road, Greenbelt, MD, USA 20771

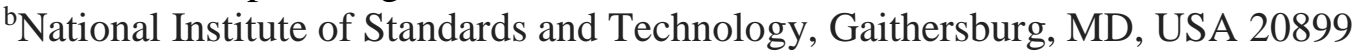 \\ 'Lambda Consulting, Harwood, MD USA 20776 \\ ${ }^{\mathrm{d} C}$ Conceptual Analytics, LLC, Glenn Dale, MD, USA 20769
}

\begin{abstract}
The slitless spectrometer plays an important role in the WFIRST mission for the survey of emission-line galaxies. This will be an unprecedented very wide field, HST quality 3D survey of emission line galaxies ${ }^{1}$. The concept of the compound grism as a slitless spectrometer has been presented previously. The presentation briefly discusses the challenges and solutions of the optical design, and recent specification updates, as well as a brief comparison between the prototype and the latest design. However, the emphasis of this paper is the progress of the grism prototype: the fabrication and test of the complicated diffractive optical elements and powered prism, as well as grism assembly alignment and testing. Especially how to use different tools and methods, such as IR phase shift and wavelength shift interferometry, to complete the element and assembly tests. The paper also presents very encouraging results from recent element tests to assembly tests. Finally we briefly touch the path forward plan to test the spectral characteristic, such as spectral resolution and response.
\end{abstract}

Keywords: grism, slitless spectrometer, high efficiency diffractive surface

\section{INTRODUCTION}

The Wide-Field Infrared Survey Telescope (WFIRST) is a NASA observatory designed to perform wide-field imaging and slitless spectroscopic surveys of the near infrared (NIR) sky for the community ${ }^{2}$. The current WFIRST design of the mission makes use of an existing $2.4 \mathrm{~m}$ diameter telescope to enhance sensitivity and imaging performance. The WFIRST payload includes two main instruments: a wide field instrument and a coronagraph instrument. The wide field instrument provides the wide-field imaging and slitless spectroscopy capability required to perform the dark energy, exoplanet microlensing, and NIR surveys. A compound grism design is selected as the slitless spectroscopy. More specifically, the slitless spectroscopy is going to survey the emission-line galaxies to cover red-shift $1<\mathrm{z}<6$ (Figure 1 ). However, the emphasis of this paper is to address how to design and build the slitless spectrometer as the part of the risk reduction of WFIRST pre-phase A study.

What distinguishes WFIRST from other previous and existing space telescopes is its large FOV, higher spectral resolution, and a faster beam. All these bring new challenges to grism optical design and fabrication. The detailed comparison, challenges, solutions, and prototype development are the discussion through this paper.

*qian.gong-1@ @asa.gov; phone 1301 286-1490; fax 1301 286-7230; nasa.gov 


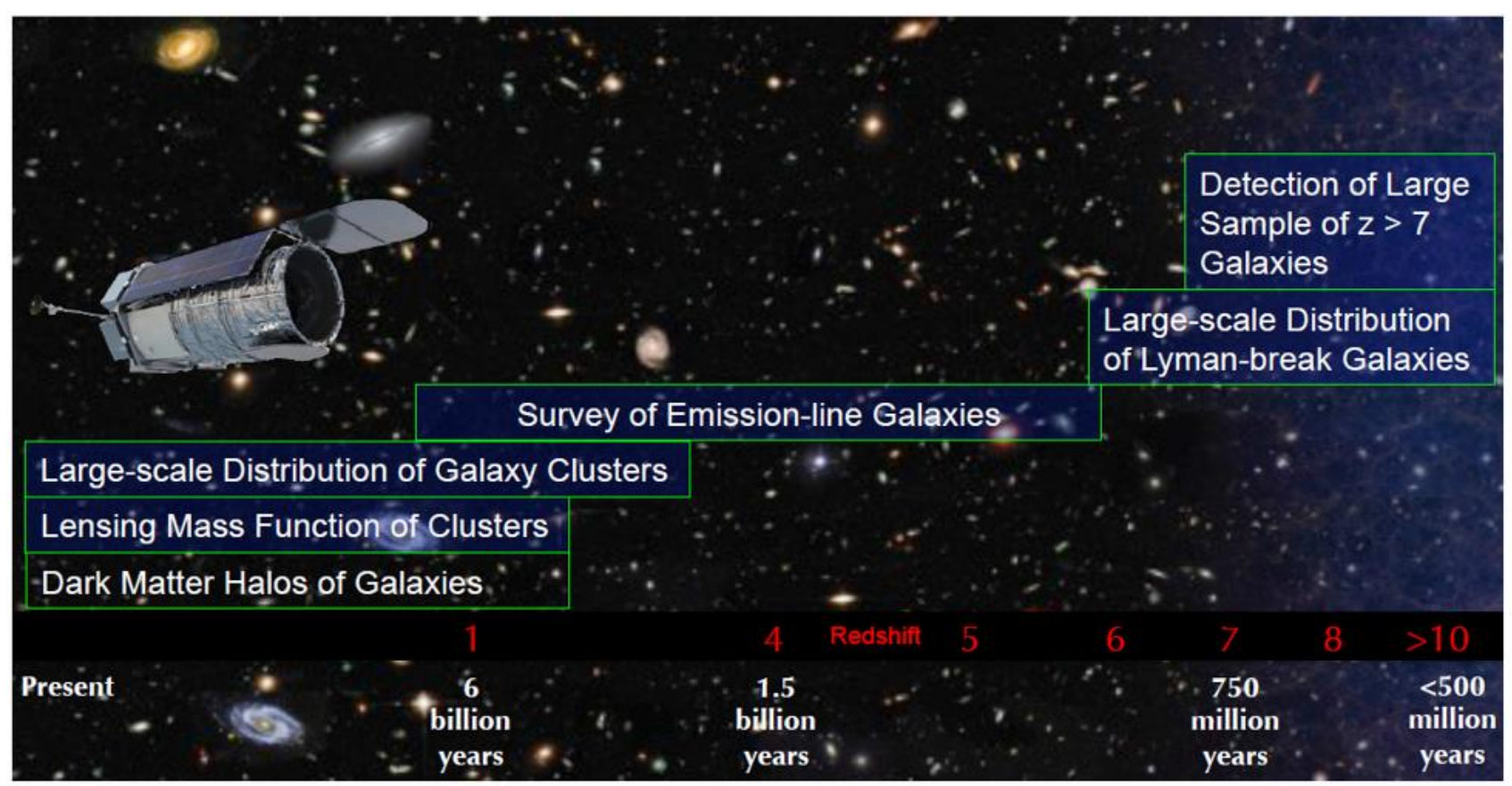

Figure 1. WFIRST-AFTA: A unique probe of cosmic structure formation history.

\section{GRISM SPECIFICATION AND CHALLENGES}

\subsection{History of Compound Grism Optical Design}

The WFIRST pre-phase A has gone through 6 study cycles. The first cycle of the compound grism design started in 2013. In the first 3 cycles, a four element compound grism was the baseline design. Almost all optical surfaces were aspheric, and the diffractive surface, grating, was on one of the aspheric surfaces. Even though selected and varying NIR glass materials were used, the image quality was still not satisfactory. So starting from cycle 4 , the addition of a second diffractive surface was introduced. The aberration created by the grating in a converging $f / 8$ beam with a large FOV was easily corrected by the diffractive corrector. This design is used for building a prototype grism assembly. The subsequent Cycle 5 grism design does not have any major change from cycle 4 . However, in the mission concept review design (MCR or "cycle 6"), the minimum wavelength has decreased from $\lambda=1.35 \mu \mathrm{m}$ to $1.0 \mu \mathrm{m}$. Meanwhile, in order to split some visible light for grism fine guidance, a dichroic beam splitter at a fairly large incidence angle has been inserted before the grism dispersion. This dichroic becomes one of the elements in the grism assembly. These changes have made the cycle 6 grism design very different than the current prototype design. In the following sections, both prototype and cycle 6 designs are discussed and compared.

\subsection{WFIRST Compound Grism Specification}

The wide-field grism assembly is to be inserted into a $\sim \mathrm{f} / 8$ beam around the pupil image plane as one element in the filter wheel of the wide-field instrument. The goal is to provide the dispersion with the spectral resolution $\mathrm{R} \sim 700$ in the wavelength range from $1.35 \mu \mathrm{m}$ to $1.95 \mu \mathrm{m}$ for prototype, and that from $1.0 \mu \mathrm{m}$ to $1.9 \mu \mathrm{m}$ for cycle 6 study. The detailed specifications for prototype and cycle 6 study are listed in Table 1. In addition to the specification, the following restrictions have to be met simultaneously.

1. When inserting the grism into the $\mathrm{f} / 8$ beam, the image plane has to be unchanged in order to be parfocal (same focal position as the filters held in the same element wheel) with other filters.

2. The position of each ray from central wavelength has to be unchanged after the dispersion. This implies that a prism has to be used to make the compound grism assembly zero deviation. 
3. Because the grism is one of the elements in the filter wheel, the available volume is very limited. The grism has to have a very compact design. For cycle 6 study, a dichroic beam splitter is inserted before the grism to reflect non-signal visible light onto 2 detector arrays for providing fine guidance during exposures. The requirements and restrictions above have made this grism design much more challenging from design to fabrication, to alignment, and to test. In section 2.3, the grism of HST wide field camera 3 will be used as an example to show why WFIRST grism is so challenging.

Table 1. Grism specification for prototype and cycle 6 design.

\begin{tabular}{|c|c|c|}
\hline & Prototype & MCR design \\
\hline Wavelength range $(\mu \mathrm{m})$ & $1.35-1.95$ & $1.0-1.9$ \\
\hline FOV $\left(^{\circ}\right)$ & $0.788 \times 0.516$ & $\begin{array}{l}\text { Overall area is unchanged } \\
\text { Shape has minor change }\end{array}$ \\
\hline Average beam diameter at grism $(\mathrm{mm})$ & 120 & 120 \\
\hline Beam f/-ratio at grism (mm) & $\sim \mathrm{f} / 8$ & $\sim \mathrm{f} / 8$ \\
\hline Wavefront error & Diffraction limited & $\begin{array}{l}\text { Diffraction limited at } \lambda>1.3 \mu \mathrm{m} \text {, Spot size } \\
\text { similar }\end{array}$ \\
\hline Spectral resolving power (per 2 pixels) & $645-900(461 \times \lambda)$ & $461-876(461 \times \lambda)$ \\
\hline Capability & Dispersion only & Dispersion and pointing for spectrometer \\
\hline Compactness & $\begin{array}{l}\text { 70mm total thickness for a fixed } \\
\text { diameter } \sim 120 \mathrm{~mm}\end{array}$ & $\begin{array}{l}\text { w/o dichroic, total thickness is } \sim 52 \mathrm{~mm} \text {, } \\
\text { with dichroic } \sim 80 \mathrm{~mm}\end{array}$ \\
\hline
\end{tabular}

\subsection{Grism Comparison: WFIRST versus HST}

Grisms have been used in a number of HST instruments, Wide Field Camera 3 (WFC3) ${ }^{3}$, Near Infrared Camera and Multi-Object Spectrometer (NICMOS) ${ }^{4}$, Advanced Camera for Surveys (ACS), etc. A single grism itself was used there. For WFIRST, it is totally different, controlling the image quality is greatly challenging even using compound grism assembly. The parameters in Table 2 shows the differences between WFIRST and HST.

Table 2. Optical system comparison between WFIRST and HST.

\begin{tabular}{|l|l|l|}
\hline & \multicolumn{1}{|c|}{ WFIRST } & \multicolumn{1}{|c|}{ HST WFC3 } \\
\hline FOV & 0.28 degree $^{2}$ & 0.0012 degree $^{2}$ \\
\hline Spectral resolving power & $\mathrm{R}=700$ & $\mathrm{R}=130$ \\
\hline $\mathrm{f} / \#$ & 8 & 11 \\
\hline
\end{tabular}

The 3 parameters listed in Table 2 are the key parameters in optical design to estimate how challenging the spectrometer optical design is. The WFIRST FOV is more than 100 times larger than HST WFC3. As we know, the off-axis aberration is a function of FOV, depending on the type of the off-axis aberration, the field dependent can be linear, quadratic, cubic, etc. For example, when the field angle increases 10 times, the astigmatism will increase 100 times! Next, if the grism is in collimated space, no aberration is created. When the f/\# gets smaller and smaller, the aberration from the dispersive element becomes more and more difficult to control. The f/\# of WFIRST is f/8, but HST WFC3 is slower at 11 . The third, as a spectrometer, the difficulty is also a function of spectral resolution. The higher of the resolution, the more difficult it 
is. Besides all this, the aberration is also a function of the aperture. The larger the aperture, the more difficult it is, even with the same f/\#. The size of the WFIRST grism $(\sim 120 \mathrm{~mm}$ in diameter) is much larger than the grism on WFC3.

\section{OPTICAL DESIGN OF GRISM ASSEMBLY}

\subsection{Optical Design of Prototype}

As mentioned above, the optical design of the prototype uses two diffractive surfaces. The reason for adding one more diffractive surface is that a grating in a non-collimated space introduces a lot of aberration. This aberration is not only a function of incident angle, but also linear to the wavelength. It is really difficult to compensate the aberration scaled to wavelength using the conventional optics, even when freeform aspheric surfaces are used. However, aberration created from diffractive surfaces is all scaled to wavelength. Based on this, adding another diffractive surface to compensate the aberration created by the grating becomes a natural solution for this grism. The additional diffractive surface is a very weak Fresnel lens like surface with non-symmetry in $\mathrm{x}$ and $\mathrm{y}$ directions. The off-axis aberration created by this surface efficiently compensate the aberration created by the grating surface, which makes the final image quality from design residual diffraction limited. With the second diffractive surface, the entire optical design has simplified. All element material is fused silica. The surfaces are either flat or spherical. The whole assembly is more compact to fit into the very limited space. Figure 2 shows the prototype grism optical design and the grism position in the WFIRST wide-field instrument.
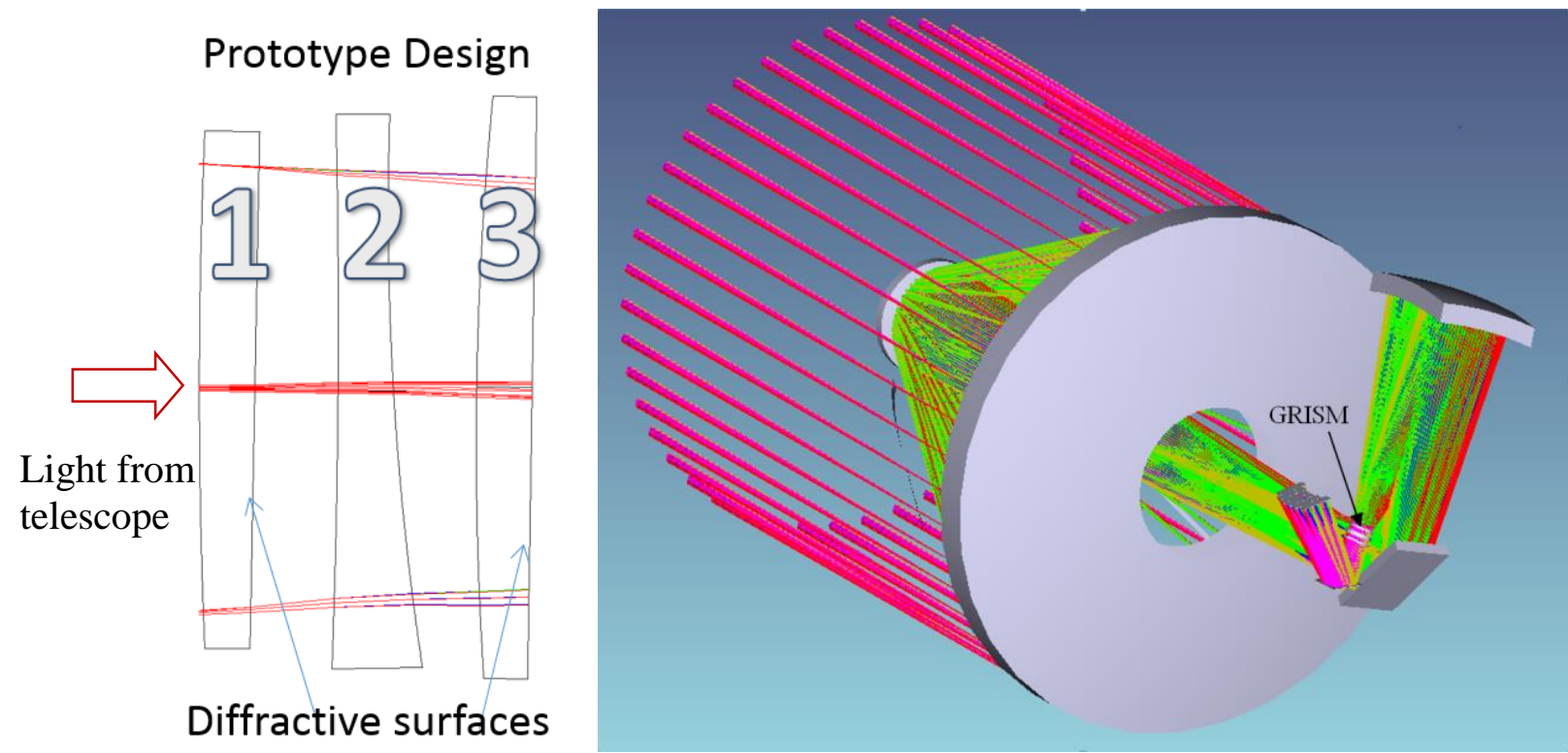

Figure 2. On the left is the prototype grism layout. On the right is the grism with the WFIRST wide-field instrument.

The main function of each element is as following: 1. Element \#1 is an aberration corrector. It is to compensate the wavelength scaled aberration created by the grating (Element \#3). 2. Element \#2 is a prism. It is to make the whole grism assembly zero deviation at the central wavelength. Both surfaces of the prism are spheres for aberration control. 3. Element 3 is the key element of the grism, it provides the required spectral dispersion $(R=461 \lambda)$. The grating in this assembly is not a regular straight line grating. It has curved lines and variable line spacing for further aberration control. The patterns of the two diffractive surfaces, the corrector and the grating, are shown in Figure 3 (a) and (b). The optimized grism design provides a diffraction limited performance for all wavelength and fields. Figure 4 shows the spot diagram of the grism design across the FOV and wavelength range meets the specification. 


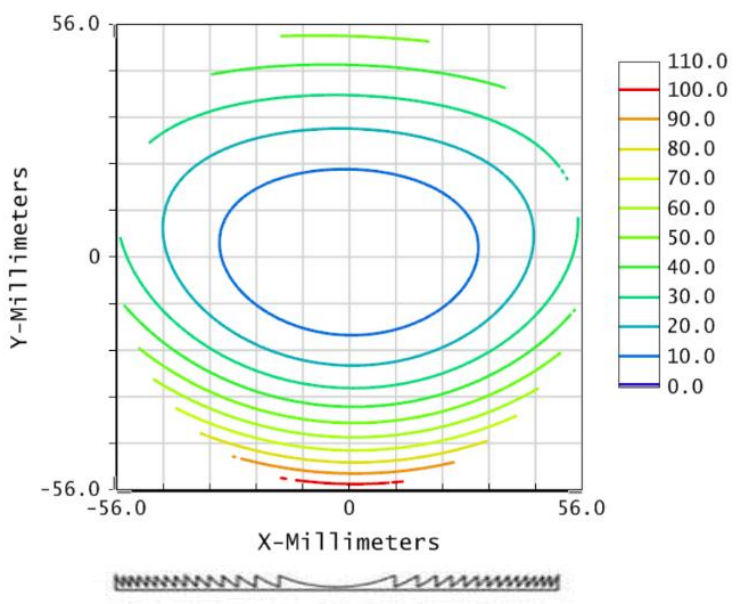

(a)

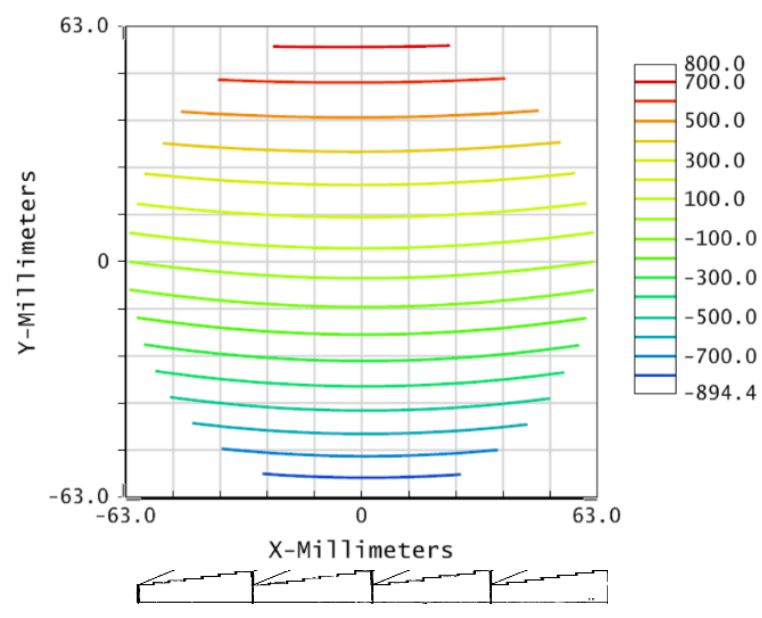

(b)

Figure 3. Diffraction patterns of Elements \#1 and \#3 diffractive surfaces. (a) is for Element \#1 which is made by grey scale process and (b) is for Element \#2 by multi-step overlay process. 


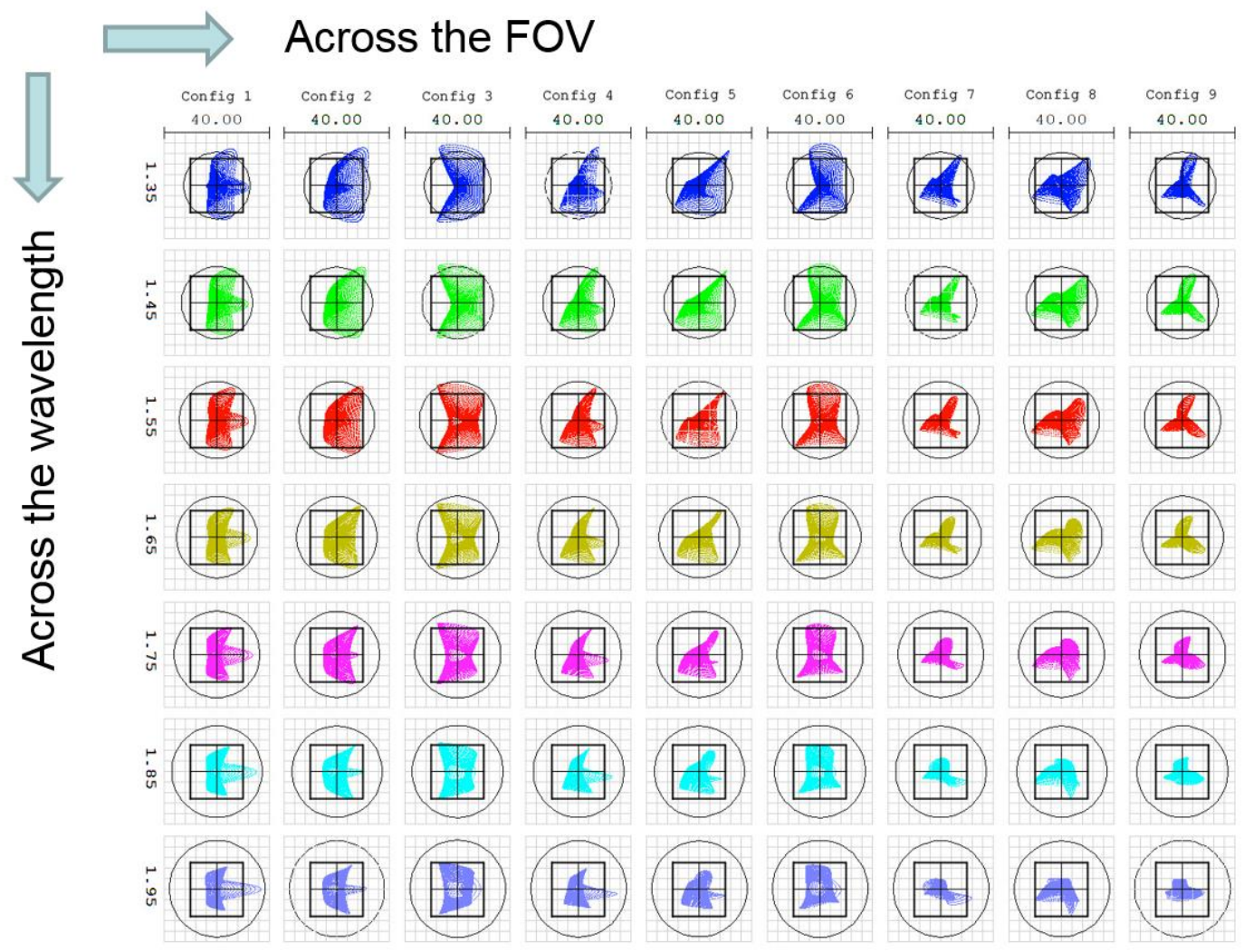

Figure 4. Diffraction limited performance for the entire FOV and entire wavelength range.

\subsection{Optical Design of Cycle 6}

The cycle 6 optical design is similar to the prototype in principle. There are two major differences. The first is the wider wavelength range. In cycle 6 the wavelength range has extended to $1.0 \mu \mathrm{m}$ to $1.9 \mu \mathrm{m}$ compared to $1.35 \mu \mathrm{m}$ to $1.95 \mu \mathrm{m}$ in prototype. The second one is that a dichroic beam splitter is added before the grism to provide a non-dispersive image for fine guidance sensor. This dichroic has to be tilted enough to avoid the reflected beam from interfering with the incoming beam and the structures in the payload. As a result, the rest of the grism elements have to be tilted too to minimize the aberration. Figure 5 shows the cycle 6 optical design and its dual functions in the WFIRST instrument.

The function of the three grism elements are similar to the prototype design. The added dichroic beam splitter reflects the visible light onto the silicon detector array to provide fine guidance for the grism assembly. The image quality of this design is similar to the cycle 5 design measured by spot size. But the wavelength range shifts to shorter wavelength, and the diffraction limited performance is scaled to wavelength, so it is not completed diffraction limited for the entire wavelength range. 


\section{Cycle 6 Design}



\section{Dichroic Diffractive surfaces}

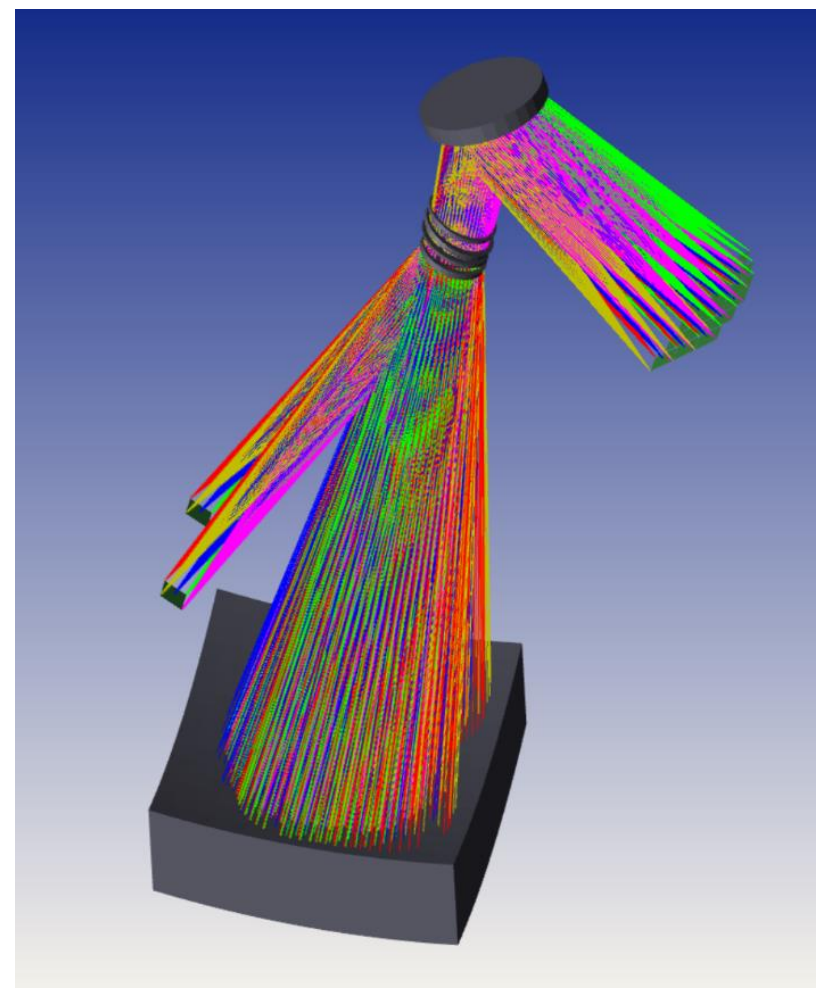

Figure 5. Cycle 6 grism optical design. On the left is the grism layout. On the right is the grism with the beam splitter to direct the visible light to guidance detectors. Ray trace from M3 is not shown for visibility.

\section{CHALLENGES AND SOLUTION FOR PROTOTYPE}

There are many challenges in the process of building the prototype. In this section, we discuss each challenge and how the challenge was solved.

\subsection{Diffraction efficiency of the DOE (Diffractive Optical Element) surfaces}

The two DOE surfaces in the design greatly improve the spectrometer performance. However, it requires high diffraction efficiency for each DOE surface in order to obtain the high throughput and reduce the ghosts from unwanted orders. To solve this problem, microlithography and reactive plasma etch (dry etch) technology was selected to make the DOE surfaces. In the optical design phase, this problem had already been considered. So fused silica material was selected for the elements with DOE surfaces. Fused silica is a dry-etch friendly material. The MEMS companies have accumulated extensive experience for dry-etching the patterns into the fused silica substrate with high efficiency close to $100 \%$ at the selected peak wavelength.

It is worth to mention that we have tried two different dry etch technologies: gray scale and multi-step overlay. For the low density frequency, $<5$ grooves / mm, either technology works. However, for the higher density, multi-step overlay yields much higher diffraction efficiency. This is because the gray scale is not able to get very sharp tops and bottoms. This is also because the slope of the gray scale is not as steep as multi-step overlay. This is also why the mechanically ruled gratings can only provide a peak efficiency of about $80 \%$.

\subsection{Dry-etch diffractive pattern onto a substrate with a wedged convex back surface}

Most of the dry etch equipment is made for semi-conductor industry to etch flat wafers. Our substrates are not only too thick (as shown in Figure 2) but also wedged with a curved back surface. We have tried two ways to solve this problem. For element \#1 with a coarse groove density, we found a vendor having a gray scale lithography equipment that can 
accommodate our thick substrate. However, a special custom mount had to be made to hold the curved back for laser writing and etching. For element \#3, the grooves are too dense for gray scale. Multi-step overlay was selected. But the equipment can only accept flat wafers less than $6 \mathrm{~mm}$ thick. So we developed an innovative way to handle it. We made two separate parts: a thin flat substrate and a thicker substrate with a convex surface. The total thickness is the same as the specified thickness for Element \#3. We first etched the diffractive pattern into the thin flat substrate, then use adhesive free bonding technology to bond them together. Elements \#1 and \#3 have been successfully been fabricated, delivered and tested at NASA Goddard.

\subsection{Complex element tests}

The element tests are also challenging. The element \#2 is a powered prism, which is the easiest of all three. Because it does not have a diffractive surface, so it can be tested at any wavelength. However, the two concave surfaces on the prism also introduce enough aberration to make the test challenge for normal interferometer test. The solution is to design a special Computer Generated Hologram $(\mathrm{CGH})^{6}$ to compensate the huge aberration when testing the powered prism using a Zygo interferometer at HeNe wavelength.

The two other elements, elements \#1 and \#3, are even more challenging due to the diffractive surface on one surface. It is known that the aberration introduced by a diffractive surface has a strong wavelength dependency, usually proportional to wavelength. Because the operation wavelength range is from $1.35 \mu \mathrm{m}$ to $1.95 \mu \mathrm{m}$, the CGH has to be designed in that wavelength range, and an IR interferometer has to be used to test them. In the relatively few IR interferometers that work in our wavelength range, $\lambda=1.55 \mu \mathrm{m}$ is popular. So the CGHs were designed for that wavelength. It is probably worth to mention that we also tried to design a CGH to test the elements at HeNe wavelength. The design itself works. However, the element's diffractive surfaces are designed and fabricated to provide highest diffraction efficiency at peak wavelength of $1.65 \mu \mathrm{m}$. The diffraction efficiency at $0.6328 \mu \mathrm{m}$ is too low to be seen. During the test, the designed first order signal is buried in ghost fringe. We have successfully designed IR CGHs and tested the elements using IR interferometer. The details will be described in the latter part of the paper.

\subsection{Mechanical mount design to minimize wavefront distortion at cryogenic temperature}

Based on the operation wavelength range and the signal to noise analysis, the grism needs to operate at temperatures from $150 \mathrm{~K}$ to $170 \mathrm{~K}$. Therefore, the mount design has to take the temperature change into account. Two approaches are used for the design: 1. The mount material is selected as Invar to best match the fused silica CTE (Coefficient of Thermal Expansion). 2. A special feature to the ring was designed to further reduce the stress at cryo. Besides the operation at cryo, the very limited volume is another challenge to the mechanical assembly design. As for solutions, low CTE Invar was selected as the mount material. Very compact mounts and assembly with special wire-EDM features are built-in to control the CTE introduced stress on elements.

\subsection{Alignment and assembly challenge}

The grism optical alignment presents another challenge to the prototype. Each element has only one axis of symmetry, which is already a challenge to the alignment. The diffractive surfaces make the process far more challenging. For this prototype, we tested the assembly based on the mechanical tolerance and fine tuning under the interferometer to see if the complicated optical alignment can be eliminated. This method was successful.

\section{GRISM OPTICAL ELEMENT TEST}

All optical elements have been completed and delivered. They are undergoing a series of tests and characterizations. The warm test is completed. The cryo test plan and setup has been prepared. However, we are still looking for a vibration insensitive IR interferometer to overcome Dewar vibration.

\subsection{Grating coupon diffraction efficient test}

In order to assure that the grating efficiency meets our prediction and support the WFIRST throughput requirement, grating samples have been designed, ordered, delivered, and characterized. A sample blazed grating using multiple overlay technology has been fabricated by JenOptik Inc. A visible grating was initially designed to allow us to use the available sources and the detector. For the NIR wavelength of the grism in $1.35 \mu \mathrm{m}-1.95 \mu \mathrm{m}$, the period is greater, the 
fabrication difficulty and scattering effect are reduced. Therefore if the high efficiency in the visible can be reached, it will not be a problem for longer wavelengths. Figure 6 shows the diffraction efficiency measurement setup.
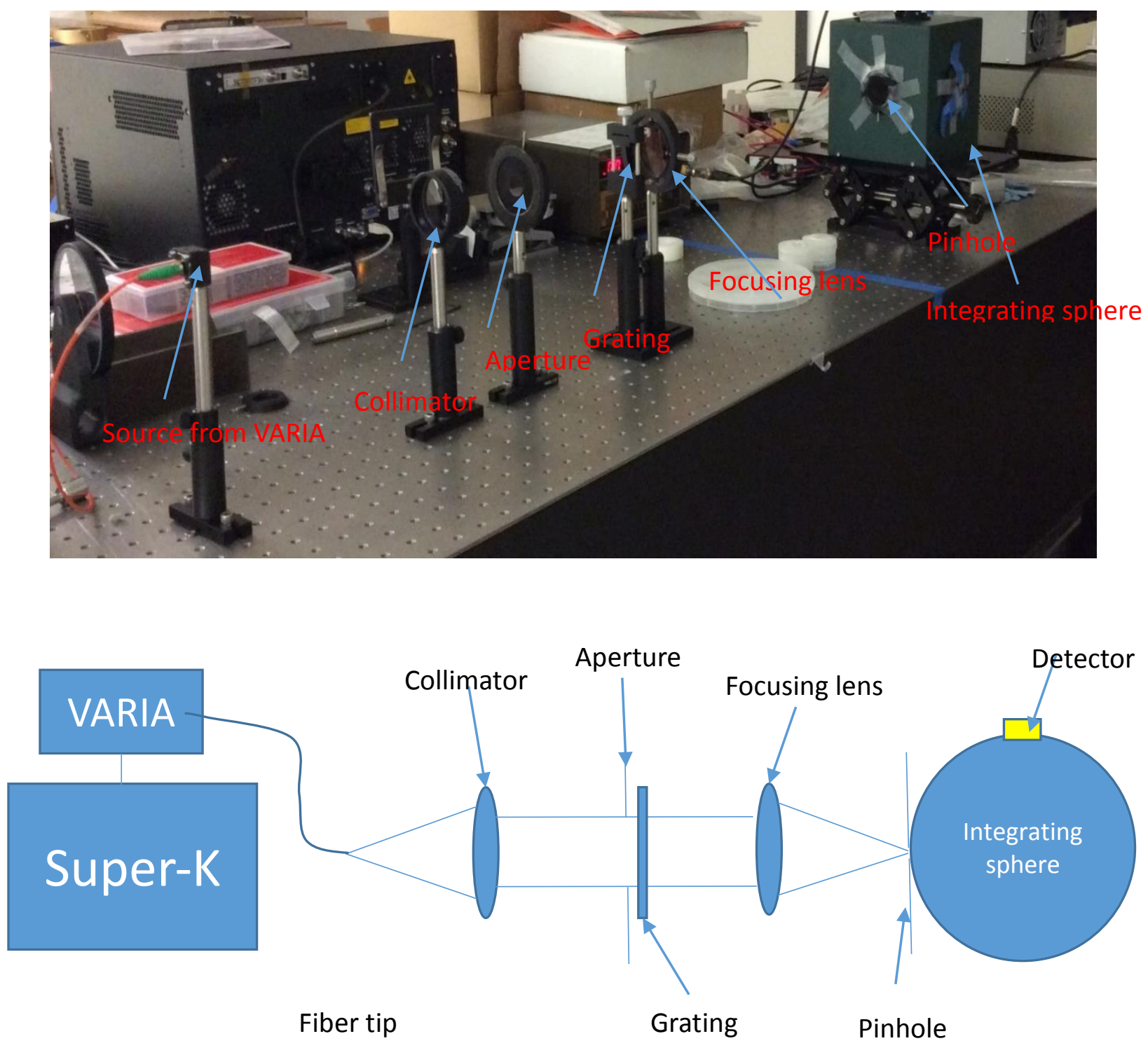

Figure 6. The photo on top is the diffraction efficiency measurement setup, At bottom is a schematic of the measurement.

A super-continuum source from NTK was selected as the light source. The light from the source is directed into VARIA, a tunable single line filter. The output of the VARIA goes to a single mode fiber with a wavelength bandwidth of $4 \mathrm{~nm}$. The tip of the fiber is positioned at the focus of a collimator. The grating is in the collimated space. An aperture is inserted in front of the grating to make sure all light go through the grating area. A focusing lens is positioned after the grating to focus the light into a pinhole at a port of an integrating sphere. The pinhole size is adjusted to pass only designed diffraction order. During the test, we adjust the central wavelength and record the output of the desired order. Then we remove the grating and let all light received by the integrating sphere. The diffraction efficiency is the ratio of the throughput from the desired grating order to the total throughput without the grating. Figure 7 shows the measured diffraction efficiency versus wavelength. 




Figure 7. Measured diffraction efficiency of the visible wavelength initial grating coupon.

The measurement wavelength starts at $480 \mathrm{~nm}$, the shortest wavelength the super-continuum source provides, and ends at $740 \mathrm{~nm}$. This range contains the wavelength with the design peak efficiency. The shape of the curve fits very well with the theoretical prediction ${ }^{5}$. The blue and red curves are measured for two different antireflection coatings. The green curve shows the difference of the two coating using the scale on the right.

Figure 8 shows a theoretical prediction of diffraction versus wavelength in the defined $1.35 \mu \mathrm{m}-1.95 \mu \mathrm{m}$ range. It is noted that the efficiency drops by about $10 \%$ for the two edge wavelengths.

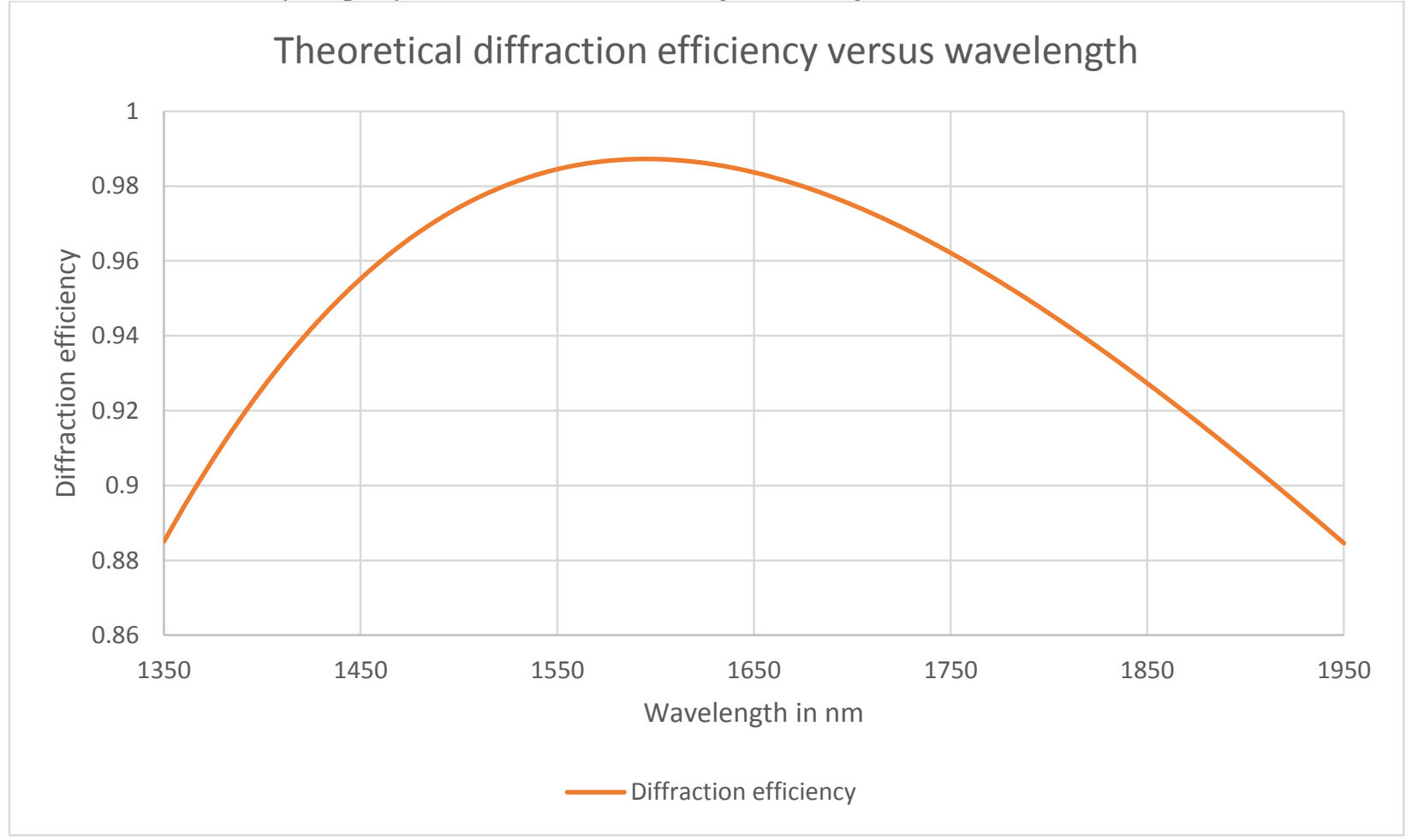


Figure 8 . Theoretical diffraction efficiency prediction based on 16 step overlay.

\subsection{Diffractive Corrector Efficiency Measurement}

The aberration corrector is a slow freeform Fresnel lens. The light from different orders can't be completely separated. This makes the efficiency measurement very difficult. Plus, the wedged convex back surface adds more aberration to make the spot from the wanted order very large. Before the measurement, we modeled it using Zemax. The result shows that even if the diffraction efficiency is not very high, the measurement still holds, by calculating spillover power in the area that other orders overlap the wanted order.

Figure 9 (a) shows the computer generated spot diagram with different orders. (b) is the image of the real measurement at $1590 \mathrm{~nm}$, and (c) is the curve indicates the measurement error with respect to element diffraction efficiency. The measurement setup is very simple. The Element \#1 was inserted to a collimated beam. An IR detector array was positioned to the focus of Element \#1. During the measurement, a special effort was made to find unwanted orders. But they are all too weak to be found, buried under the detector array noise. This indicates that the diffraction efficiency of this diffractive aberration corrector is high, On the other hand, it implies that when working together with other grism elements, the wanted order forms a tiny spot on the final WFIRST detector array, the ghost from unwanted orders will be way below the detector noise.



(a)

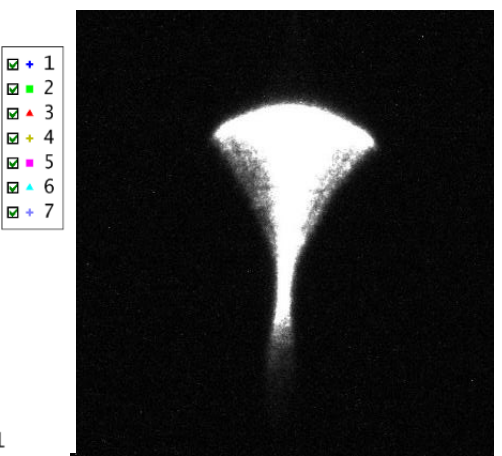

(b)

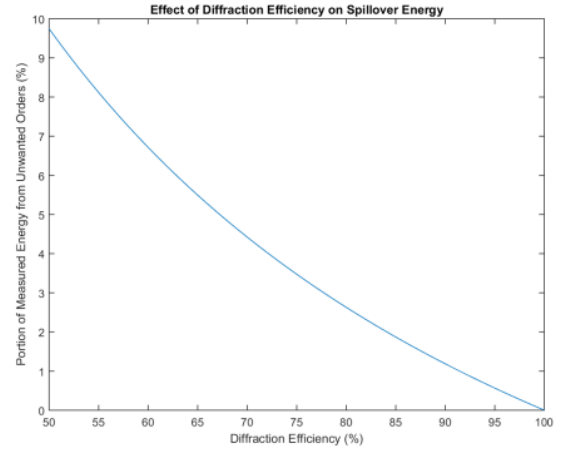

(c)

Figure 8. (a) Spots from all diffraction orders from -3 to +3 . $1^{\text {st }}$ order (wanted order) is in pink. (b) Image of the only observed $1^{\text {st }}$ order spot. (c) Measurement error versus diffraction efficiency.

\subsection{Grism Elements Test}

\subsubsection{Element \#1: Aberration corrector}

As mentioned in Section 3, the element \#1 is to correct the aberration created by the grating in the $f / 8$ space. The first surface is a flat with a diffractive pattern etched into the surface. The second surface is a convex, but the axis of the vertex is tilted relative to the first surface. After a market survey, we realized that only one company provided a quotation based on best effort. Fortunately, this best effort turned out to be good enough. The measured results show that both diffraction efficiency and wavefront are good. Figure 10 shows the photo of the mounted grism elements, Element \#1 is on the right. 


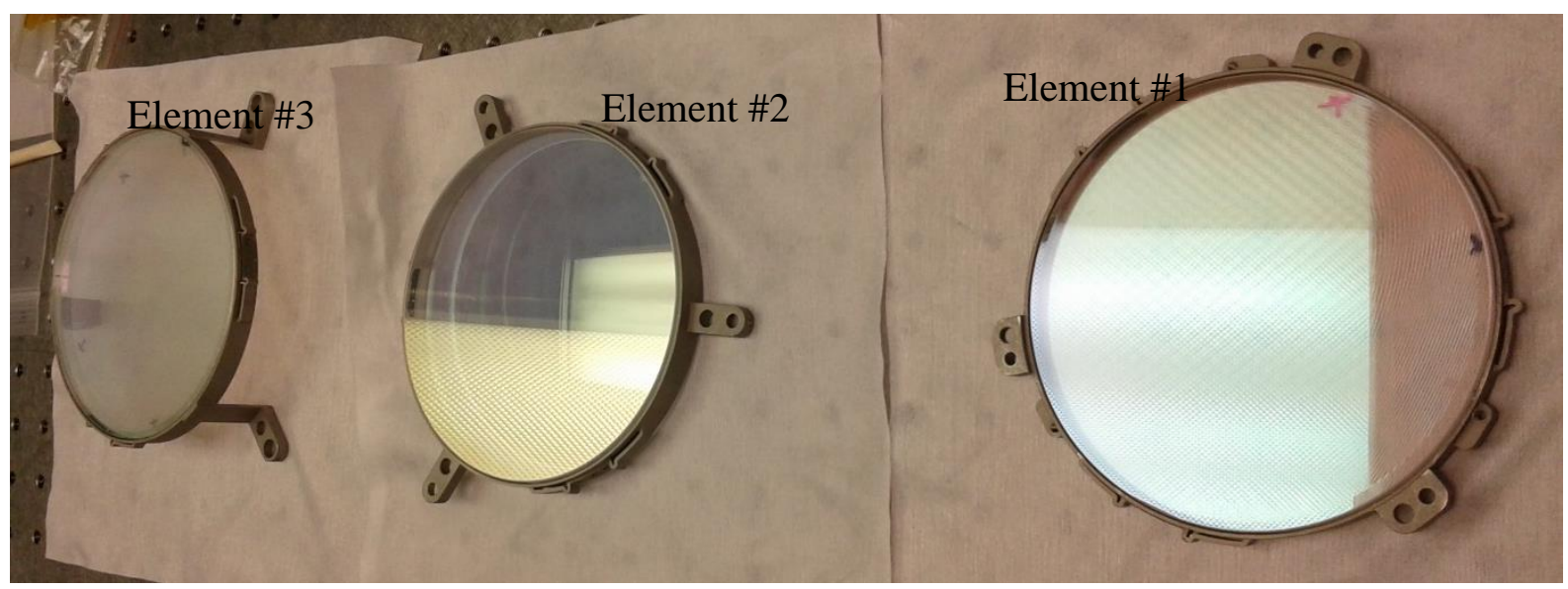

Figure 10. Photo of completed and mounted prototype grism elements \#1, \#2, and \#3.

The wavefront measurement was designed using an interferometer with a specially designed CGH. Based on the available IR interferometer wavelength, the CGH is designed for $\lambda=1.55 \mu \mathrm{m}$. We started with a visible CGH design, but the unwanted diffraction orders dominate the light returned to the interferometer. No meaningful interferogram was observed. It also needs to mention that the $\mathrm{CGH}$ needs to be a phase $\mathrm{CGH}$ in order to obtain a good fringe contrast in the interferogram, though it does not have to be blazed. Figure 11 shows the designed measurement setup. It is seen from Figure 11 that the interferometer has a collimated beam output beam. The CGH sits in the collimated space. The advantage of having the $\mathrm{CGH}$ in collimated space is that the CGH displacement relative to the interferometer is not sensitive. When we design the CGH, we have in mind to use the CGH for both ambient and cryo test. The elements in cryo Dewar usually move a little from ambient to cryo, so we could compensate the motion by moving the CGH accordingly. The focus is used as a carrier for this CGH design. It matches the weak power of the Element \#1 perfectly, so the beam after the Element \#1 is collimated again. Only a flat mirror is needed to return the beam back to the interferometer. In this case, the mirror displacement is not sensitive either, only tip/tilt angle is critical. The angular adjustment is straight forward under the guidance from interferogram fringes. Figure 12 shows the pattern of the CGH. The existing non-symmetry is to compensate the aberration introduced by the wedge of Element \#1. Because of the existing central obscuration in the Element \#1, the central portion of CGH is used as a metrology pattern to align the Element \#1 relative to the interferometer.

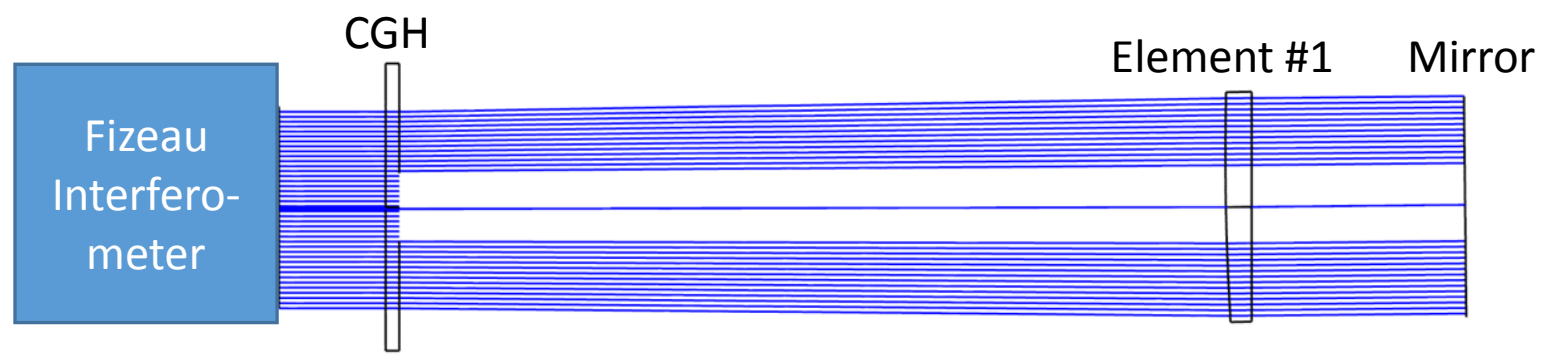

Figure 11. Layout of designed Element \#1 wavefront measurement. The focus carrier of the CGH is designed to match the power of Element \#1 to make the beam at the return mirror collimated. 




Figure 12. Hologram pattern of CGH for Element \#1.

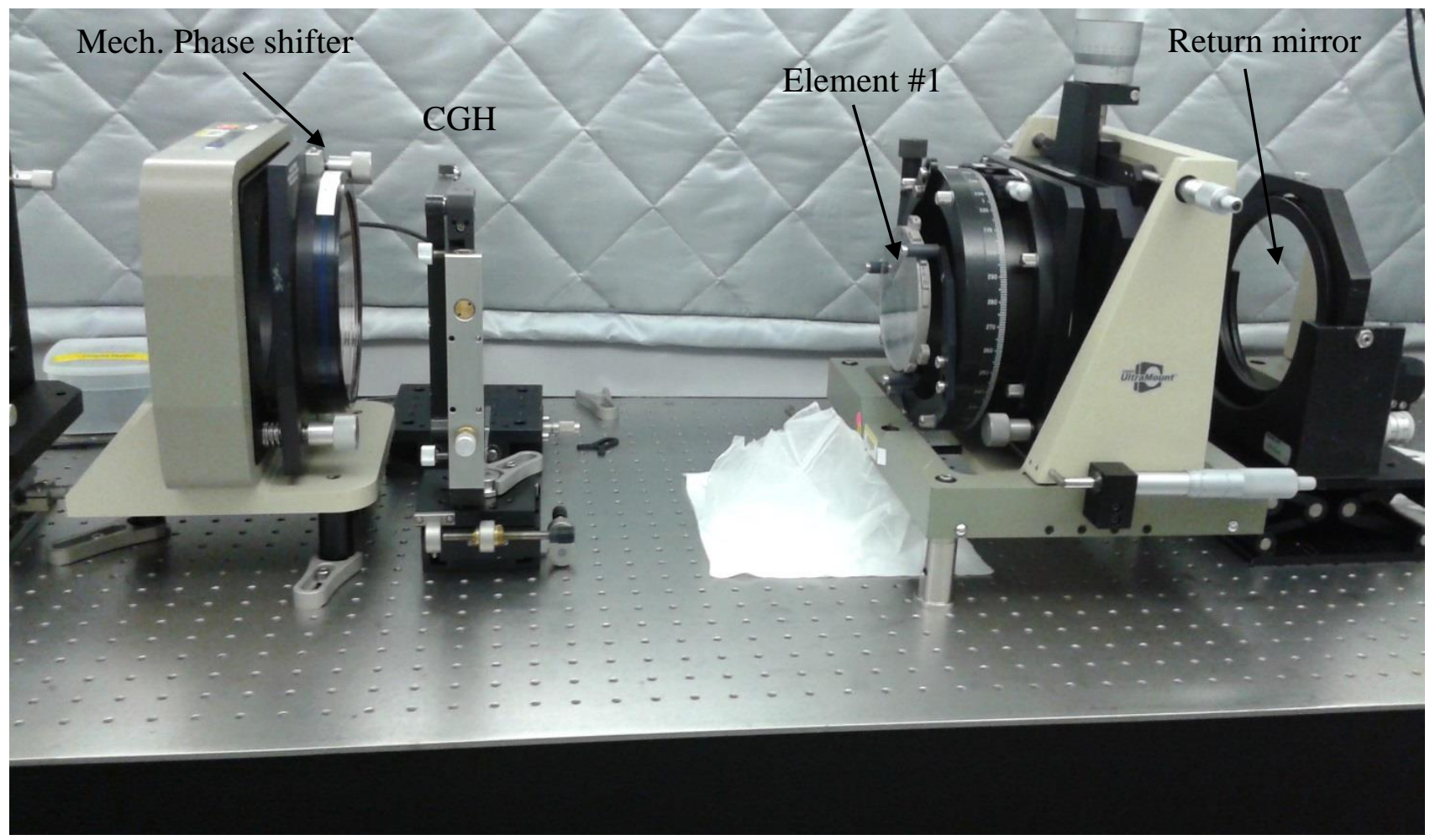

Figure 13. Element \#1 test setup in the NIST IR ${ }^{3}$ infrared interferometer. This setup was used for both mechanical phase shift and wavelength phase shift measurements.

The transmitted wavefront measurements for Elements \#1 and \#3 were made with both piezo-mechanical and wavelength phase shifting interferometry to have greater confidence in the measurement results. The mesurements were made using two different interferometers, the improved infrared interferometer $\left(\mathrm{IR}^{3}\right)$ at NIST and a Zygo interferometer at NASA. The $\mathrm{IR}^{3}$ interferometer was originally developed for thickness measurements of silicon wafers ${ }^{8}$. For the measurements of the WFIRST elements the interferometer was modified with a new collimator lens and a piezo-mechanical phase shifter. The interferometer light source is a single-mode tunable diode laser with a wavelength range centered at $1550 \mathrm{~nm}$. The piezomechanical shifter had a range of about $420^{\circ}$ at $1550 \mathrm{~nm}$, sufficient for basic phase shifting algorithms. We chose a phase 
shifting algorithm with 7 samples and $60^{\circ}$ phase shift between samples for the measurements with mechanical phase shifting. For the measurements with wavelength phase shifting we used a phase shifting algorithm with 13 samples and $60^{\circ}$ phase increment between samples. Due to the long interferometer cavity the wavelength change required to effect the $4 \pi$ phase shift was very small. Based on the specifications of the diode laser and the operating parameters used we estimate that the wavelength change during a phase measurement was approximately $0.01 \mathrm{~nm}$.

The collimated test beam from the interferometer is first incident on a transmission flat (with flatness error $\lambda / 30$ at 633 $\mathrm{nm}$ ) that is mounted on the mechanical phase shifter. The transmission flat can be seen on the letft in Figure 13. Because the CGH is in a collimated space, a transmission flat can be inserted with negligible additional aberration. When the beam passes the CGH, it slowly diverges to match the power of Element \#1, meanwhile the same amount of aberration with opposite sign is generated to cancel the aberration of Element \#1. In addition to the measurements made with the IR ${ }^{3}$ interferometer, the same measurement was also performed using a IR Zygo interferometer at GSFC with wavelength shifting only. The tested wavefront errors from all three measurements are listed in Table 3. Figure 14 is one of the wavefront maps measured by $\mathrm{IR}^{3}$ phase shift interferometer, but analyzed with Zygo software. The test result shows that the Element \#1 meets the design requirements.

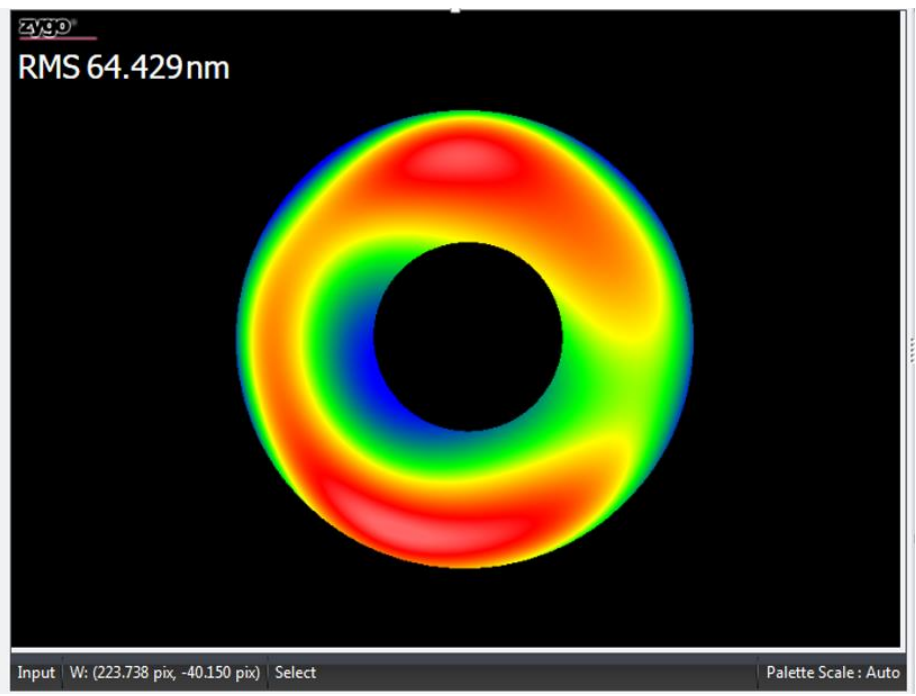

Figure 14. Zernike polynomial fit to a wavefront map of Element \#1 that is measured by NIST IR ${ }^{3}$ phase shift interferometer. The raw wavefront data is then processed using Zygo software MX.

Table 3. Measured Element \#1 wavefront error using 3 different means.

\begin{tabular}{|l|l|l|l|}
\hline & NIST IR ${ }^{3}$ piezo shifting & NIST IR $^{3}$ wavelength shifting & Zygo wavelength shifting \\
\hline Wavefront error (RMS) & $64 \mathrm{~nm}$ & $64 \mathrm{~nm}$ & $86 \mathrm{~nm}$ \\
\hline
\end{tabular}

The data in Table 3 show that the results from the mechanical phase shifting and wavelength shifting at NIST are identical. This demonstates that the wavelength shift for our long optical path length works just as good as phase shift. The wavelength change during measurements with wavelength shifting does not cause significant measurement errors. The wavefront error from Zygo measurement is a little different, which might be largely because the measured wavelength of Zygo IR interferometer is $1.5445 \mu \mathrm{m}$ that offs a little from the claimed $1.55 \mu \mathrm{m}$. But the CGH is designed for $1.55 \mu \mathrm{m}$.

\subsubsection{Element \#2: Wedged Prism}

Element \#2 is a wedged prism with two concave surfaces . It is the easiest test among the three grism elements, because it does not include any diffractive surfaces. However, a CGH is still needed for correcting the huge aberration by tilted spherical surfaces. The setup for this CGH design has some similarities as Element \#1. The CGH is in collimated space and the focus is used as a carrier. The main difference is that Element $\# 2$ test setup is designed in visible at $\lambda=$ $632.8 \mathrm{~nm}$. Because no diffractive surface involved, use a HeNe interferometer is more convenient and provides more precise wavefront measurement. The mounted Element \#2 is show in Figure 10 in section 5.3.1. Figure 15 shows the Element \#2 test setup design and Figure 16 is the photo of lab setup. 


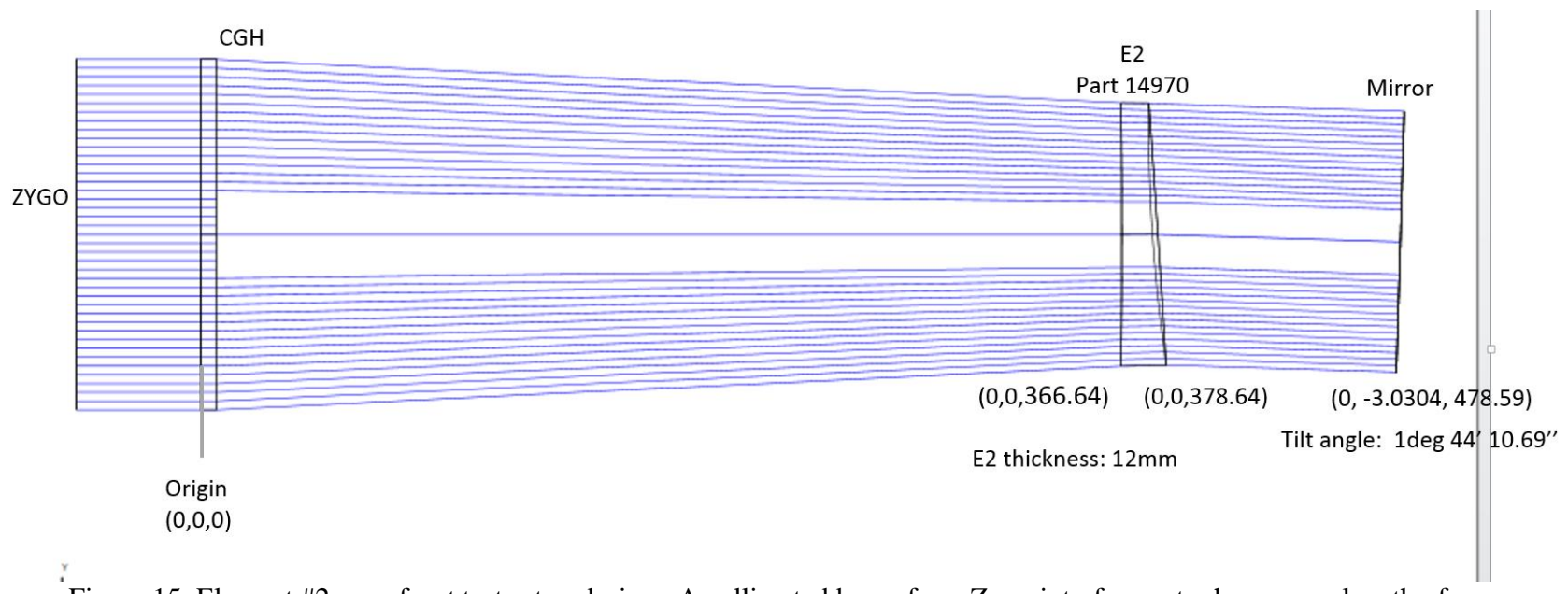

Figure 15. Element \#2 wavefront test setup design. A collimated beam from Zygo interferometer has a wavelength of $632.8 \mathrm{~nm}$. The CGH sits in the collimated space. The focus carrier converges the beam after the CGH to compensate the negative power of E2, and provide wavefront correction at the same time. The light coming out of the E2 is collimated again but slightly tilted. The return mirror is aligned nominal to the beam for returning the beam back to interferometer.

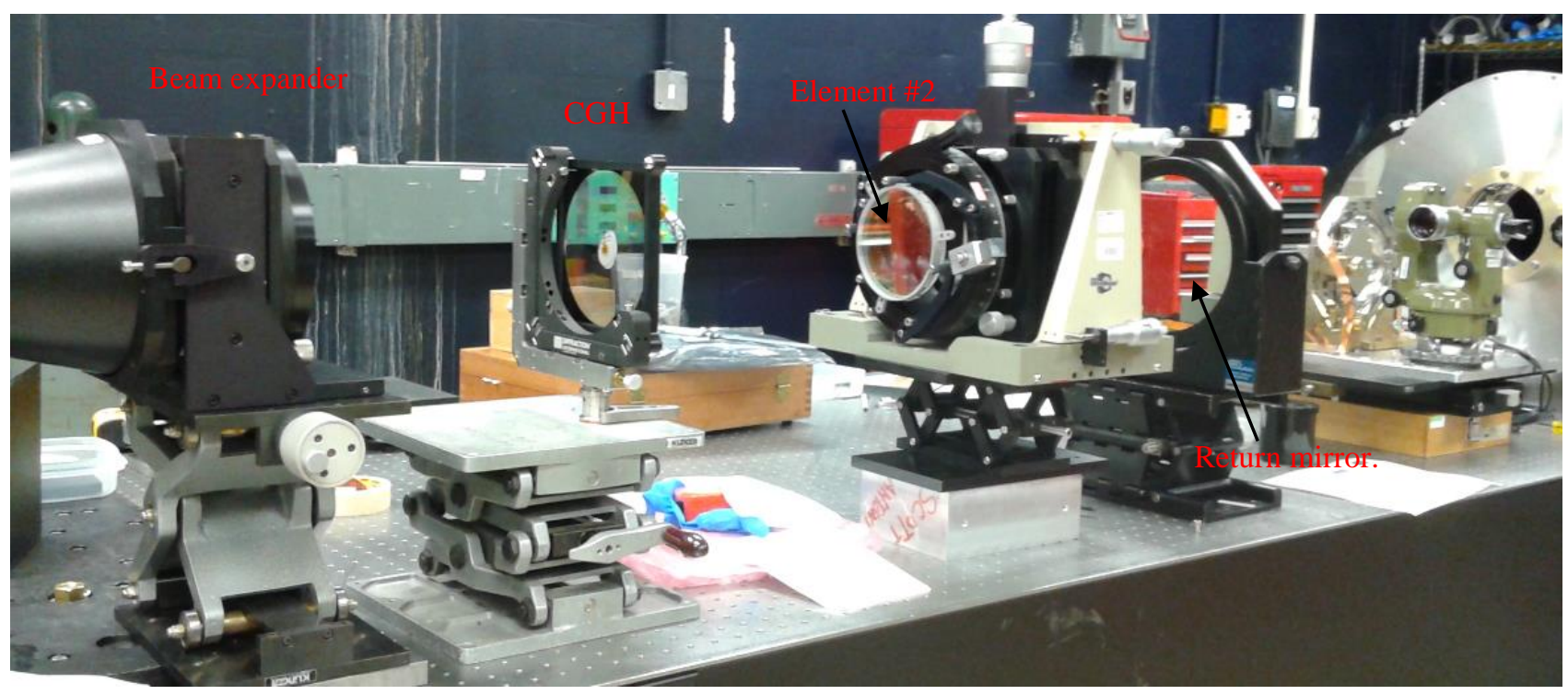

Figure 16. Real test setup of Figure 15. The theodolite behind the return mirror was used to parts setup. 


\begin{tabular}{|c|c|c|}
\hline Seidels & & \\
\hline Tilulag (Z) & 0.187 & $\lambda$ \\
\hline TritAng (Z) & -40.40 & - \\
\hline FodVlag (Z) & -0.289 & $\lambda$ \\
\hline Asthog (Z) & 0.217 & $\lambda$ \\
\hline Astang (Z) & -72.07 & $=$ \\
\hline Comllag (z) & 0.280 & $\lambda$ \\
\hline ComAng (Z) & 139.60 & $=$ \\
\hline SA $(Z)$ & 0.299 & $\lambda$ \\
\hline
\end{tabular}

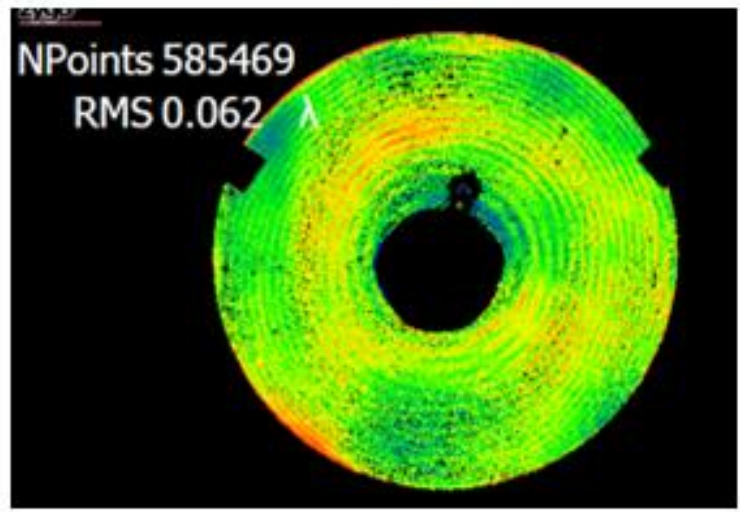

\section{Measured WF RMS: $39.2336 \mathrm{~nm}$}

Figure 17. Measured wavefront error is $<40 \mathrm{~nm}$ RMS. It meets the requirement.

Figure 17 is the measured wavefront error from the test setup in Figure xxx. The total wavefront error is $39.2 \mathrm{~nm}$, which meets our error budget. For this particular element, we also performed cryo test at $170^{\circ} \mathrm{K}$ to verify the stress induced by the mounting ring. The test result is positive. The details will be published in a different paper.

\subsubsection{Element \#3: Grating}

The grating is the last element to be tested. Its picture is shown in Figure 10 in section 5.3.1. The test setup is very similar to Element \#1. Again, the CGH is placed behind a transmission flat into the collimated test beam. The CGH is designed to ensure that the beam is collimated and aberration free after Element \#3, the wavelength of the interferometer is $1.55 \mu \mathrm{m}$. Figure 18 shows the measured wavefront map. Again, it was measured in three different ways: using the NIST IR ${ }^{3}$ interferometer with mechanical phase shifting and wavelength shifting, and a Zygo IR interferometer with wavelength shifting only. The measurement result is shown in Table 4.

Table 4. Measured Element \#3 wavefront error with standard uncertainty using 3 different methods.

\begin{tabular}{|l|l|l|l|}
\hline & NIST IR ${ }^{3}$ piezo-shifting & NIST IR ${ }^{3}$ wavelength shifting & Zygo wavelength shifting \\
\hline Wavefront error (RMS) & $69 \mathrm{~nm} \pm 1 \mathrm{~nm}$ & $68 \mathrm{~nm} \pm 1 \mathrm{~nm}$ & $55.3 \mathrm{~nm}$ \\
\hline
\end{tabular}

Finally, we would like to note that all three grism elements have acceptable wavefront error. We also like to note that all CGHs used for grism element tests are designed and built in-house. The NanoFab at NISTs Center for Nanoscale Science and Technology was used to plasma etch the amplitude CGH into phase CGH. This has not only greatly shortened test turnaround time, but also provides much more flexibility to accommodate our existing facility and equipment.

\section{GRISM MECHANICAL DESIGN AND ASSEMBLY}

The mounts manufactured to hold the grism elements were made of invar. This material was selected to minimize the wavefront distortion at $170^{\circ} \mathrm{K}$, the operational temperature of the instrument.

Each individual element was mounted onto a ring structure which had three flexure points which made contact with the optic. To mount the elements epoxy Hysol EA 9309.2 was placed on each flexure and was left to cure for 5 days while the optic rested on a Teflon mount. Afterwards, these three ring mounts were mounted onto a cell which had nominal pin 
positions that allowed to position the elements in a nominal location. However, to mount the cell onto a tip/tilt mount for measurement purposes, a triangular integration deck was used, which is shown in Figure 19(b).

The individual ring structure mechanical design was previously tested in a similar environment for another project which also had to control the CTE mismatch which can introduce wavefront degradation.

Five degrees of freedom were designed into two of the three elements to provide some adjustment once mounted and assembled. Once assembled, the need to for adjusting the third element occurred, and parts were modified to accommodate from this.

To test the performance of the mount on the elements, Element \#2 after mounting was cycled twice from $270^{\circ} \mathrm{K}$ to $160^{\circ} \mathrm{K}$. During the cryogenic cycles one of the surfaces of Element \#2 was measured in reflection through a window. After the cycles were completed, the measured wavefront was mostly undistorted, notifying a change of approximately $15 \mathrm{~nm}$ in wavefront rms error, proving that the mount and epoxy cause low distortion. The CAD model of the grism prototype with its flexures is shown in Figure 19(a).

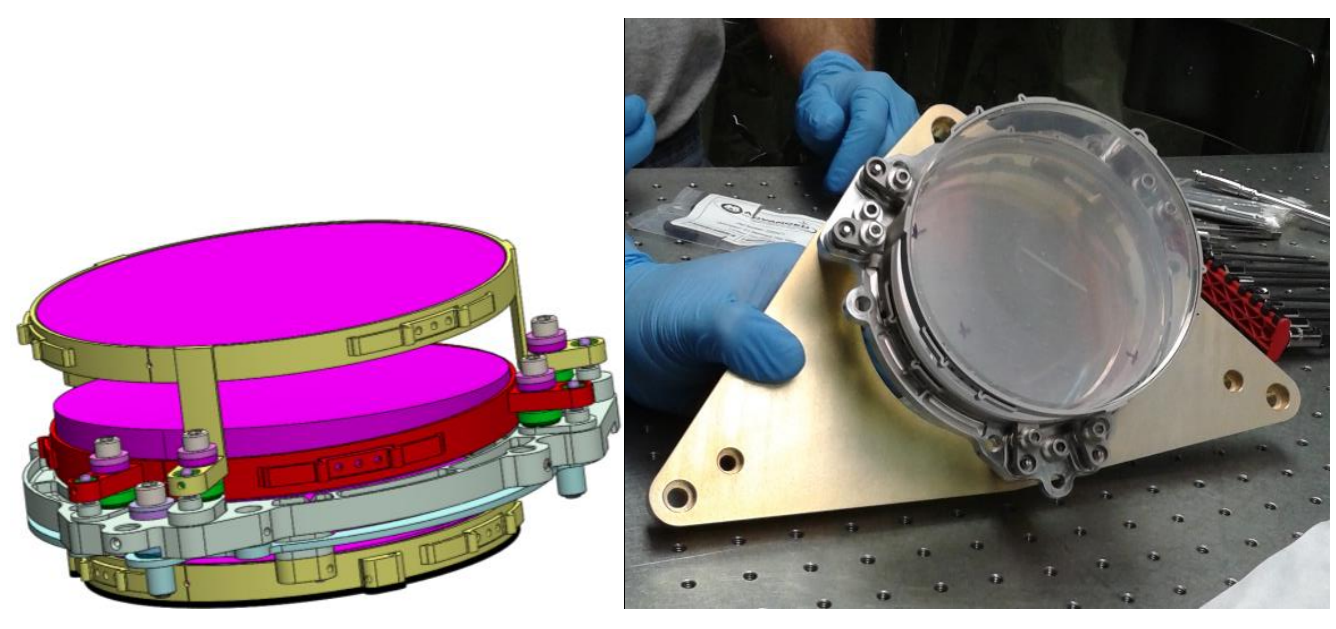

(a)

(b)

Figure 19. (a) CAD model of the GRISM assembly in its invar mounts. (b) Assembled grism prototype on integration deck.

\section{GRISM ASEMBLY TEST AND PRELIMINARY RESULTS}

After the elements were assembled, as Figure 19(b) shows, they were mounted onto the tip/tilt mount, the assembly was then placed on a hexapod in front of an infrared interferometer in non-collimated space, similar to the actual configuration of the grism in the instrument.

The testing configuration consisted of a wavelength shifting infrared interferometer, with a $1544.5 \mathrm{~nm}$ wavelength (measured with a wavelength meter), an f/7.2 transmission sphere, the grism assembly and a spherical mirror that would allow to double pass through the grism (shown from left to right in Figure 20). The setup initially started by aligning the sphere to the interferometer and then introducing the grism in the path, since the assembly does not have power. As mentioned in the previous section the mount had some degrees of freedom that would allow for clocking ( 0.4deg) and decenter adjustment ( 650microns), which proved to be helpful since the alignment of the elements is sensitive to the clocking orientation one element has with respect to the other. After doing a sensitivity analysis, the elements were positioned at optimized locations which improved the wavefront measurement. The entire assembly was placed on a hexapod that allowed easy and accurate movement of the grism when varying the different field positions. 


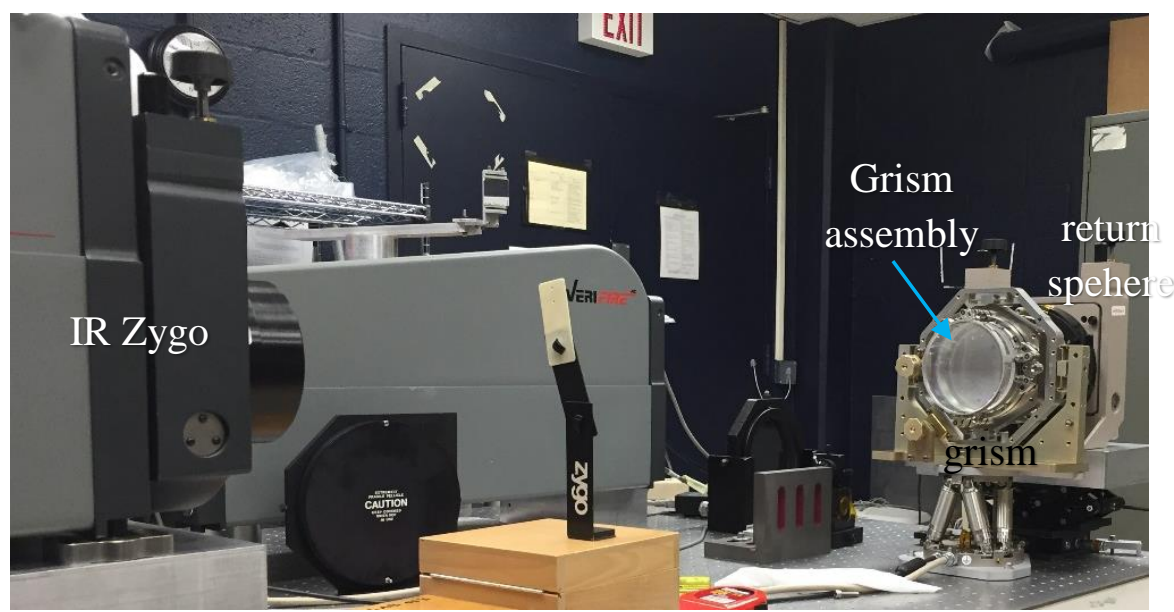

Figure 20. Test layout of the grism assembly in front of an IR interferometer with a flag indicating the focus position of the transmission sphere.

The preliminary unprocessed result of the aligned grism assembly is $~ 90 \mathrm{~nm}$ WF rms at the on-axis field position. The wavefront is shown in Figure 21(a). The dark portion in the center of this figure shows significant drop out due to the grating of Element \#1 that prints through the interferogram. However the processed measurement result (shown on Figure 21(b)) still validates the performance of the grism because the actual instrument has a pupil mask in front of the assembly that blocks out the center portion of it anyway. To obtain the processed wavefront, the high frequency errors were removed, the power term was removed also and inner and outer masks were used.

It is important to note that these results are preliminary and are only for the on-axis field position, the off-axis positions still have to be measured.

According to preliminary Zemax models which included the measured performance of each of the independent elements, the calculated wavefront performance should be $<100 \mathrm{~nm}$ rms. The allotted budget for the prototype assembly is $\sim 115 \mathrm{~nm}$ rms. Additional measurements, modeling and analysis are still required, however, these results are preliminary but encouraging.

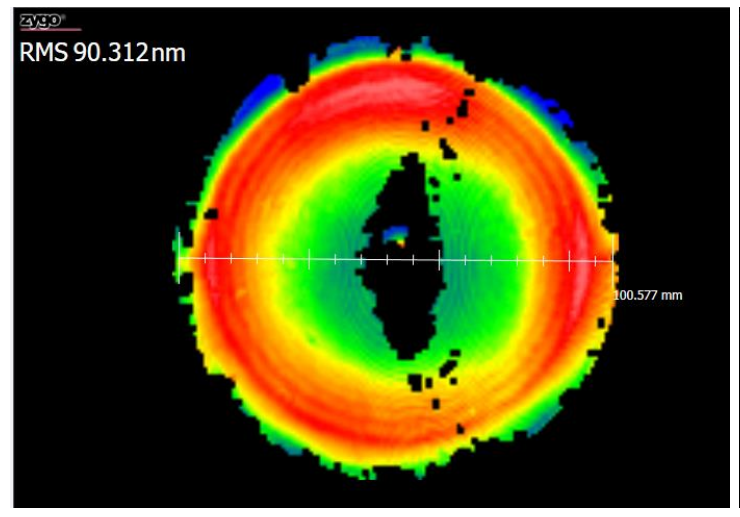

(a)

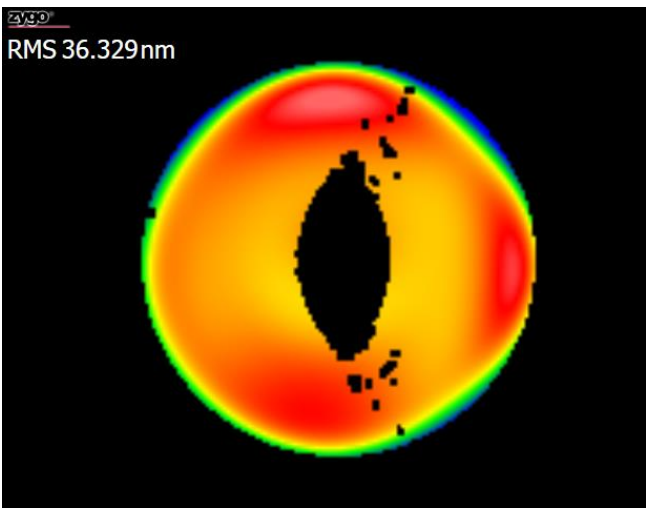

(b)

Figure 21. (a) Raw measurement of the grism assembly. (b) Processed wavefront of the grism assembly.

\section{CONCLUSION}

We have successfully designed, fabricated, assembled, and tested the cycle 5 prototype grism assembly. To date, we have verified the wavefront of each element and whole grism assembly. All elements meet specification. We are really happy to report that the performance of whole assembly at the only field position we have measured is exceed 
specification. Besides the wavefront error, the diffraction efficiency from both Element \#1 and Element \#3 has almost reached theoretical prediction. This implies that the ghost from unwanted order is minimal. Our high fringe contrast during the interferometer measurement indicates the same result. Our plan next is to build a single field WFIRST simulator to perform the spectral test: to verify spectral resolution, grism spectral response (related to diffraction efficiency versus wavelength), as well as ghost from unwanted orders. The simulator moves to different positions and tilted to corresponding angles for mapping the FOV. Based on the experience and lesson learned from the prototype development, we'll start the phase A slitless spectrometer design study soon.

Disclaimer: The full description of the procedures used in this paper requires the identification of certain commercial products and their suppliers. The inclusion of such information should in no way be construed as indicating that such products or suppliers are endorsed by NASA or NIST, or are recommended by NASA or NIST, or that they are necessarily the best materials or suppliers for the purposes described.

\section{ACKNOWLEDGMENTS}

Our research was in part performed using resources provided by the NanoFab of the NIST Center of Nanoscale Science and Technology.

\section{REFERENCES}

[1] Scoville, N. etc. "The Cosmic Evolution Survey (COSMOS) - Overview”, December, 2006 $\langle$ http://arxiv.org/pdf/astro-ph/0612305.pdf>

[2] Gehrels, N., Spergel, D., Melton, M., and Grady, K., "WFIRST-AFTA Science Definition Team Final Report”, (13 February, 2015)

[3] "Wide Field Camera 3 Instrument Handbook", Space Telescope Science Institute, January 2016 $\langle$ http://www.stsci.edu/hst/wfc3/documents/handbooks/currentIHB/wfc3_ihb.pdf >

[4] "Near Infrared Camera and Multi-Object Spectrometer Instrument Handbook", Space Telescope Science Institute, http://www.stsci.edu/hst/nicmos/documents/handbooks/current_NEW/toc.html

[5] Swanson, G. J., "Binary Optics Technology: Theoretical Limits on the Diffraction Efficiency of Multilevel Diffraction Optical Elements", MIT Lincoln Lab Technical report, Pg. 11, (1 march, 1991)

[6] Malacara, D. [Optical Shop Testing], Wiley-Interscience, A John Wiley \& Sons, Inc., Publication, third edition, 474488 (2007)

[7] Malacara, D. [Optical Shop Testing], Wiley-Interscience, A John Wiley \& Sons, Inc., Publication, third edition, 547666 (2007)

[8] U. Griesmann, Q. Wang, M. Tricard, M. Dumas, and C. Hall, "Manufacture and Metrology of $300 \mathrm{~mm}$ silicon wafers with Ultralow Thickness Variation", AIP Conf. Proc. 93, 105-110, (2007) 